\title{
Bounds and Error Bounds for unsolvable CT Markov Chains
}

\author{
Nico M. van Dijk ${ }^{1}$ \\ ${ }^{1}$ Faculty of Electrical Engineering, Mathematics and Computer Science, Department of Applied Mathematics, \\ University of Twente, Netherlands. \\ ${ }^{a)}$ n.m.vandijk@utwente.nl
}

\begin{abstract}
An approach is presented to compare two Markov Chains, particularly Continuous-Time Markov Chains (CTMC) such as to model Queueing Networks (QN). Here one may typically think of one CTMC or QN to be a solvable modification (e.g. a product form QN) of the other one, say the original, which is of practical interest but unsolvable. The approach is essentially based upon evaluating performance measures by cumulative reward structures and analytically bounding so-called bias-terms, also known as relative gains or fundamental matrix elements. A general comparison and error bound result will be provided. The approach, referred to as Markov Reward approach, is related to Stochastic Dynamic programming and
\end{abstract}

- $\quad$ may lead to analytic error bounds for the discrepancy, and

- $\quad$ may still apply while stochastic comparison fails

To motivate and illustrate the approach, the presentation will contain an instructive finite tandem queue example and a practical result for a real-life application of an Operation Theater-Intensive care unit system. Some remaining questions for research will be addressed briefly.

\section{Introduction}

\section{Motivation}

Queueing networks, as wide application area of CTMC's, are characterized by special transition structures. Unfortunately, the analytic solvability, such as for so-called product form results, of these structures typically breaks down at boundaries, such as due to finite capacity constraints. By artificially modifying the transition rates at these boundaries, such as to ensure that the global balance equations can be decomposed into more detailed solvable ones, solvability might be regained. This in turn might already be practical so as to provide simple computational approximations or bounds for a performance measure. An example of an Operating Theatre - Intensive Care Unit system will be given later on. It will thus be of interest whether the effect of the modification can be predicted and estimated analytically. To do so, a general result will first be presented to compare two CTMC's.

\section{Model description}

For the purpose of short presentation, let us directly restrict to the setting of Continuous-time Markov Chains (CTMC's). Consider a CTMC with countable state space $\mathrm{S}$ and transition rate matrix $\mathbf{Q}=q(i, j)$, with $q(i, j)$ the transition rate for a change from a state $i$ into a state $j \neq i$. For convenience, this chain is assumed to be uniformizable. That is, for some finite constant $H<\infty$

$$
\sum_{j \neq i} q(i, j) \leq H \quad(\text { for all } i \text { in } S)
$$

By virtue of the boundedness (uniformization) assumption (1), it is then well known that the CTMC can also be evaluated as a discrete-time Markov chain (DTMC) with one-step transition matrix $\mathbf{P}$, with $h=1 / H: \mathbf{P}=I+h \mathbf{Q}$; hence, with one-step transition probabilities: 


$$
\mathbf{P}(i, j)= \begin{cases}h q(i, j), & , j \neq i \\ 1-h \sum_{j \neq i} q(i, j) & , j=i .\end{cases}
$$

For some given reward rate function $\mathbf{r}$ consider the expected cumulative reward up to time $t$ as given by:

$$
\mathbf{V}_{t}(i)=\int_{0}^{t}\left(\mathbf{T}_{s} \mathbf{r}\right)(i) d s \quad \text { with } \quad \mathbf{T}_{s} \mathbf{r}(i)=\sum_{j} \mathbf{P}_{s}(i, j) r(j) .
$$

where $\mathbf{P}_{s}(i, j)$ denotes the transition probability for a transition from state $i$ into state $j$ over time $s$.

Then, under natural ergodicity and irreducibility conditions and for some given appropriate reward rate function $\mathbf{r}(i)$, i.e. $\mathbf{r}$ per unit time whenever the system is in state $i$, we are interested in an average reward or steady-state performance measure $\mathbf{G}$ :

$$
\left\{\begin{array}{l}
\mathbf{G} \text { : the expected average reward per unit time in steady state situation as can also be represented as } \\
\mathbf{G}=\lim _{t \rightarrow \infty} \frac{1}{t} \mathbf{V}_{t}(i)=\lim _{t \rightarrow \infty} \frac{1}{t} \int_{0}^{t} \mathbf{T}_{s} r(i) d s \quad \text { (for any } i \text { in } S \text { ) }
\end{array}\right.
$$

G can then be regarded as a scalar. For example, with the CTMC representing an $\mathrm{M}|\mathrm{M}| \mathrm{N} \mid \mathrm{N}$ queue with server rate $\mu$ and $n$ the number of jobs, it represents

$$
\mathbf{G}= \begin{cases}\text { Mean queue length for } & \mathbf{r}(n)=n \\ \text { Loss probability for } & \mathbf{r}(n)=\mathbb{1}_{(n=N)}, \\ \text { Throughput for } & \mathbf{r}(n)=n \mu \mathbb{1}_{(n>0)} .\end{cases}
$$

\section{MAIN RESULT}

As motivated above, now suppose that we like to compare a performance measure

$\mathbf{G}$ for an original CTMC with transition rates $q(i, j)$

with $\overline{\mathbf{G}}$ for a modified CTMC with transition rates $\bar{q}(i, j)$

say $S \supseteq \bar{S}$, for both of which the uniformization condition (1) holds with same constant $H$, and where $\mathbf{G}$ and $\overline{\mathbf{G}}$ are the average expected rewards with reward rates $\mathbf{r}$ and $\overline{\mathbf{r}}$ respectively. Here one may typically think the two CTMC's to be related in that the transition rates from one, say the modified one, can be seen as a modification of the other, say the original.

The following comparison and error bound result can now be concluded (from [1]) for comparing $\mathbf{G}$ and $\overline{\mathbf{G}}$.

It can be given in various versions. The present form, however, is most practical in the natural situation that the steady-state distribution of one of the two models, typically the modified one, is known as easily computable. Herein, let $\mathbf{V}_{k}(i)$ represent the cumulative expected reward over $k$ steps of the DTMC as by (2) when starting in state $i$ at time 0 by $\mathbf{V}_{0}=0$ and with $h=1 / H$ :

$$
\mathbf{V}_{k}(i)=h \sum_{m=0}^{k-1} \mathbf{P}^{m} \mathbf{r}(i)=h \mathbf{r}(i)+\sum_{j} \mathbf{P}(i, j) \mathbf{V}_{k-1}(j)
$$

Result 1 (Comparison and Error bound). Suppose that for $\mathbf{V}_{k}$ defined by (4) and for some nonnegative function $\beta(\cdot)$ at $\bar{S}$, all $i \in \bar{S}$ and $k \geq 0$ :

$$
0 \leq[\overline{\mathbf{r}}(i)-\mathbf{r}(i)]+\sum_{j}[\bar{q}(i, j)-q(i, j)]\left[\mathbf{V}_{k}(j)-\mathbf{V}_{k}(i)\right] \leq \beta(i) .
$$

Then with $\bar{\pi}$ the stationary distribution: 


$$
0 \leq \overline{\mathbf{G}}-\mathbf{G} \leq \sum_{j} \bar{\pi}(i) \beta(i)=\bar{\pi} \beta
$$

Remarks 1 In most of the applications, unless restrictive stochastic comparison conditions can be verified as for standard $\mathrm{M}|\mathrm{M}| \mathrm{s}$ type queueing systems, the comparison and error bound results generally go hand in hand. That is, in order to establish a comparison result by verifying the left inequality from (5) one will necessarily also have to verify the right inequality from (5), which itself might lead to an error bound as formulated in the result. The comparison result is of interest by itself as it may lead to a performance upper or lower bound or both for a specific performance measure of interest. This might even apply when the system itself violates stochastic monotonicy properties (e.g. [2]).

2 (Error bounds). Result 1 may lead to small error bounds in either of two ways:

(i) When either the difference between the transition rates $q$ and $\bar{q}$ is small, uniformly in all states. Here one may typically think of small perturbations or inaccuracies in system parameters.

(ii) When the transition rates $q$ and $\bar{q}$ may differ quite strongly in specific states $i$, but where the likelihood of being in such states is rather small. Here, one could typically think of a system modification or truncation.

3 (Bounded bias-terms). A crucial step to verify (either inequality) from (6) is to bound the difference terms (in stochastic dynamic programming also known as relative gain or bias-terms) of the form:

$$
\mathbf{V}_{k}(j)-\mathbf{V}_{k}(i) .
$$

Clearly, the cumulative reward function $\mathbf{V}_{k}$ will generally grow linearly in $k$ and thus be unbounded over time $k$. However, for fixed $i, j$ the difference terms as in (7) can generally be bounded uniformly over all $k$. more accurate analytic bounds for (7) might even be established in an analytic manner by induction by using the dynamic reward relation (4).

\section{Application: An Operating Theatre-Intensive Care Unit (OT-ICU)}

As a specific application the estimation of the congestion probability for an Intensive Care Unit (ICU) system can be assisted by a secure computation. As the ICU involve intensified care for post-operative patients, that is after surgery has taken place and while an operated patient will be kept on hold when no ICU bed is available, an interaction between the operating theatre (OT) and the ICU will have to be incorporated. This in turn leads to tandem queueing structures which, beyond exponentiality assumptions, are known to be hard to solve analytically. However, as lives can be at risk, a secure and proper dimensioning of ICU systems should have to take place.

Let $c$ be the number of ICU beds. By applying result 1 the following (possibly counter-intuitive) result can then be proven (see [3]), as based on the triple steps below. These steps can be seen as generic for unsolvable (e.g. typically due to finite capacity constraints) Queuing Network applications

\section{(Generic steps)}

1. A modification of the original CTMC (QN) into a solvable (typically of product form type ) CTMC ( $Q N)$,

2. A comparison of the the unsolvable and solvable system based on comparing their transitions (rates),

3. A technical verification of condition (6) by inductively bounding, by (4), the bias terms (7).

\section{Result 2 (OT-ICU sytem)}

With $c$ the number of ICU beds and $B$ the steady state probability for the ICU to be congested, that is the long run time fraction that all $c$ ICU-beds are occupied, and $\mathbf{E}(c)$ : Erlang's loss expression for an $\mathrm{M}|\mathrm{M}| \mathrm{c} \mid \mathrm{c}$ queue with arrival rate $\lambda$ and service rate $\mu$ per server, where $\rho=\lambda / \mu$ :

$$
\mathbf{E}(c)=\frac{\rho^{c}}{c !}\left[\sum_{k=0}^{c} \frac{\rho^{k}}{k !}\right]^{-1},
$$

we have

$$
\mathbf{E}(c) \leq B \leq \mathbf{E}(c-1)
$$

Here $\mathbf{E}(c-1)$ is Erlang's loss expression (8) with $c$ replaced by $c-1$. 
Intuitively, the value $\mathbf{E}(c)$ can well be expected to be a reasonable approximation, as if the ICU can be regarded in isolation. Indeed, it accurately appears so. At sample path basis, though, one may easily construct numerical counter (intuitive) examples. A proof for $\mathbf{E}(c)$ to be a sharp but strict lower bound appears far more difficult (see [3]). Clearly, for practical purpose the far more inaccurate but secure upper bound seems more useful.

Real-life numerical support shows that these bounds (particularly the upper bound) can be most useful for dimensioning ICU's, such that a secure congestion probability bound is guaranteed, e.g. of at most $\mathbf{1 0 , 5 , 2}$ or $\mathbf{1 \%}$. As an additional appealing feature, these bounds can be expected to be insensitive, i.e. not to depend on the exponential property of ICU-sojourn times, and therefore be most practical. A formal proof is still open.

\section{CONCLUSIONS}

Other applications include for example more complex networks of Erlang loss structures ([4]), the estimation of overflow or loss probabilities in overflow structures ([5]), the truncation or expansion of Jackson Networks ([6]), or extensions to non-exponential queueing systems ([7]). Several questions and other extensions remain of research interest.

\section{REFERENCES}

[1] N. M. Van Dijk, "Error bounds and comparison results: the Markov reward approach for queueing networks," in Queueing Networks (Springer, 2011), pp. 397-459.

[2] P. G. Taylor and N. M. Van Dijk, "Strong stochastic bounds for the stationary distribution of a class of multicomponent performability models," Operations Research 46, 665-674 (1998).

[3] N. M. van Dijk and N. Kortbeek, "Erlang loss bounds for OT-ICU systems," Queueing Systems 63, 253-280 (2009).

[4] R. J. Boucherie and N. M. van Dijk, "Monotonicity and error bounds for networks of Erlang loss queues," Queueing systems 62, 159-193 (2009).

[5] N. M. van Dijk and E. van der Sluis, "Call packing bound for overflow loss systems," Performance Evaluation 66, 1-20 (2009).

[6] N. M. van Dijk, "Error bounds for state space truncation of finite Jackson networks," European Journal of Operational Research 186, 164-181 (2008).

[7] N. M. Van Dijk and M. Miyazawa, "Error bounds for perturbing nonexponential queues," Mathematics of Operations Research 29, 525-558 (2004).

[8] N. M. Van Dijk and M. L. Puterman, "Perturbation theory for Markov reward processes with applications to queueing systems,” Advances in Applied Probability 20, 79-98 (1988). 